\title{
Introducción de marca de banano orgánico en el mercado ecuatoriano
}

\section{Introduction of organic banana brand in the Ecuadorian market}

Víctor Alexander Villanueva Cevallos

Cristhian Daniel Añazco Correa

Lorenzo Bonisoli

Universidad Técnica de Machala, Ecuador

Autor por correspondencia: vvillanueva_est@utmachala.edu.ec

Fecha de recepción: 12 de agosto del 2019 - Fecha de aceptación: 13 de diciembre del 2019

\section{Resumen}

El objetivo del estudio es proponer un plan de marketing para introducir una marca de banano orgánico. La fruta tiene un potencial de crecimiento de consumo. El artículo se centra en proponer bases para las asociaciones o empresas bananeras en cuanto a la comercialización del producto. Se aplicó una metodología de tipo transversal, con trabajo de campo y utilización de instrumentos como encuestas, entrevistas, observación directa. El público objetivo que se ha identificado son mayoritariamente mujeres entre los 20 a 40 años de edad, con remuneración mensual superior a un salario básico unificado, pertenecientes a la clase social media-alta, que cuidan del bienestar propio; tendientes al consumo de alimentos saludables que contribuyan a la sostenibilidad medioambiental. Con respecto al mix del marketing se formularon estrategias de packaging, etiquetado, propuesta de valor; se estimó el precio en base a los costos de la competencia; se propone un canal corto entre productor, mayorista y cliente final; los medios publicitarios definidos son offline y online, garantizando el impacto y visibilidad del producto en el mercado.

Palabras claves: análisis del entorno; banano orgánico; público objetivo; marketing mix

\begin{abstract}
The purpose of the study is to propose a marketing plan to introduce an organic banana brand. The fruit has a potential for consumption growth. The article focuses on proposing bases for banana associations or companies in terms of product marketing. A cross-sectional methodology was applied, with field work and use of instruments such as surveys, interviews, direct observation. The target audience that has been identified are mostly women between the ages of 20 and 40, with monthly salaries exceeding a unified basic salary, belonging to the upper-middle social class, who take care of their own well-being; aimed at the consumption of healthy foods that contribute to environmental sustainability. Regarding the marketing mix, packaging, labeling, value proposition strategies were formulated; the price was estimated based on the costs of the competition; a short channel is proposed between producer, wholesaler and final customer; The defined advertising media are offline and online, guaranteeing the impact and visibility of the product in the market.
\end{abstract}

Key words: environment analysis; organic banana; target; marketing mix 


\section{Introducción}

Actualmente, a nivel mundial ha incrementado la demanda de productos orgánicos en un $10 \%$ con respecto a los de origen convencional. (Méndez, 2015). Una probable explicación de este fenómeno se puede encontrar en los diferentes problemas de salud ocasionados en la última década por alimentos tratados con químicos y en particular en los que contienen plomo y mercurio. Por esta razón, el producto orgánico representa para los consumidores una alternativa más saludable y ecológicamente sostenible respecto del producto convencional (Tambaco, Alarcón y Alarcón, 2019).

La creciente demanda de producto orgánico ha generado que la extensión de suelo fértil destinado para el cultivo orgánico en 2015 ha correspondido a 50.9 millones hectáreas a nivel global, con un crecimiento de 6.5 millones al respecto del año anterior. Además, en los años siguientes esta cifra tuvo aumento exponencial llegando a un total de 97.7 millones de hectáreas considerando los cultivos en áreas agrícolas y silvestre (Acosta, López y Coronel, 2018).

El producto orgánico aportar beneficios de distintos puntos de vista, no solamente a nivel de impacto sobre la salud de las personas y el medioambiente, también en garantizar una cadena de comercialización más justa, donde hay preferencias para los proveedores, la fuerza de trabajo local y los insumos locales, hacen énfasis en el respecto de los derechos laborales de los trabajadores y se desarrolla una más igualitaria distribución de los recursos económicos (Andrade y Ayaviri, 2018). La producción orgánica se concentra en la utilización responsable de los recursos naturales básicos; en la minimización de la producción de desechos y la correspondiente maximización del uso de material reusable y reciclable en equilibrio entre la unidad productiva y el ecosistema en donde la actividad se desarrolla (Alves y Pacheco, 2015).

A nivel mundial existen 178 países en los cuales se desarrolla la actividad orgánica; de estos Ecuador con un total de suelo cultivado con producto orgánico de 39.824 hectáreas, que representa el $0.7 \%$ de la totalidad (Wille y Lernoud, 2018). La producción orgánica ecuatoriana se concentra en los principales cinco productos agrícolas destinados a la exportación: el banano, el cacao, el café, la caña de azúcar y la fruta tropical. Sin embargo, el banano es el principal producto orgánico producido en país.

La producción bananera ecuatoriana es el principal rubro económico y la principal exportación no petrolero por generación de ingresos y de trabajo. Las áreas a mayor producción bananera corresponden a las provincias de Guayas, Los Ríos y El Oro (Zambrano, Berrezueta y García, 2017). Según el modelo agroexportador de la agricultura ecuatoriana la parte prominente de la producción orgánica está destinada para la exportación, mientras es mínimo el consumo de banano orgánico local, ya que generalmente a nivel interno se destina el banano que se considera de rechazo, debido a que no cumple con los estándares estéticos de exportación a pesar que la calidad de la fruta sea análoga a la exportada (MAG, 2017).

La provincia de El Oro presenta un número relativamente bajo de productores que se dedican a la producción de banano orgánico. Un total de 162.039 Ha. De superficie cultivada de banano solo el 12\% corresponden a plantaciones orgánicas (Estrada y Encalada, 2018). En la 
ciudad de Machala el banano orgánico es producido por pequeños agricultores, cuyas fincas presentan condiciones favorables para la producción, en general se reúnen en asociaciones y gremios para poder gozar de una fuerza de negociación más importante al respecto de los exportadores internacionales (García, Santillán, Blancas y Valenzuela, 2012).

El objetivo del presente trabajo es presentar un plan de marketing que pueda ser utilizado por un gremio de productores de banano orgánico de la provincia de El Oro. De hecho, la posibilidad por los pequeños productores de abrir un canal de comercialización interno permitiría de un lado una fuente de ingresos extra y por otro lado la posibilidad de proveer a los consumidores locales un producto de calidad superior.

El estudio tiene la siguiente organización: En primer lugar es necesario explorar por medio de datos secundarios el comportamiento de las personas en cuanto a preferencia de productos orgánicos y el posicionamiento actual del mismo. Se analiza el mercado actual, desde el micro y macro entorno, permitiendo establecer el punto de partida para el estudio; se profundiza la investigación de tipo transversal, mediante la aplicación de encuestas, entrevistas y observación cuyos resultados guiaron las estrategias de mercadeo en cuanto a producto, precio, plaza, promoción; se detalla el plan de acción y presupuesto estimado con las tácticas, responsables, tiempos, recursos para su posible ejecución, finalmente podemos hacer lectura de las conclusiones determinadas en los hallazgos investigativos.

\section{Marco teórico}

El análisis del marco teórico se desarrolla de forma dual, de hecho, en primera instancia se presenta el análisis del macro entorno empresarial y en un segundo momento el análisis de la industria de la producción y comercialización bananera. Cabe mencionar que el análisis de los datos se derivó de información secundaria y primaria con trabajo de campo utilizando las herramientas de encuestas, entrevistas y observación directa, aplicados en los principales supermercados de Machala. De esta manera, se fundamenta la parte complementaria del estudio que fueron las propuestas de estrategias de marketing para el banano orgánico.

\section{Análisis del macro entorno}

Para determinar las amenazas y oportunidades que se generan en el entorno de la empresa, se utiliza el análisis que evalúa los aspectos políticos, económico, social y tecnológico (Lamas, Brasil, Correia, Picanço y Navarro, 2017). Estas fuerzas del macro entorno al ser ajenas a la empresa se convierten en factores incontrolables (Torrabadella y Saiz, 2016).

\section{Político}

El Ecuador ha desarrollado políticas básicas ambientales, en las cuales establece como principio fundamental, promover el desarrollo sostenible a nivel económico como ambiental. De la misma manera el permitir la regeneración natural de los ecosistemas a través del cumplimiento del objetivo 3 del plan nacional de desarrollo, garantizando la sostenibilidad ambiental (SNI, 2019). 
En legislación tributaria el Estado Ecuatoriano, debe acatar impuestos entre ellos el Servicio de Rentas Internas, de las actividades únicas del sector bananero, es decir, ingresos originarios de la producción y venta local, de acuerdo a la Ley Orgánica de Régimen Tributario Interno (SRI, 2019).

La producción orgánica en Ecuador se rige a los lineamientos planteados por la Ley orgánica del régimen de la soberanía alimentaria, cuyo objetivo es garantizar a los pueblos y comunidades el acceso a alimentos sanos y culturalmente apropiados Art.1; en la misma línea, el Art. 14. Fomento de la producción agroecológica y orgánica indica que el estado estimulará la producción mediante capacitaciones, líneas de crédito, nexos de comercialización en mercados internos y externos. Los productos certificados son una forma posible de obtener y mejorar la sostenibilidad (Bonisoli, 2016).

Reglamento de la normativa de la producción orgánica agropecuaria en el Ecuador (Acuerdo No. 302); Art. 1. Aborda las normas y procedimientos para producción, elaboración, empaque, etiquetado, almacenamiento, transporte, comercialización, la exportación e importación de productos orgánicos; asegurar que la cadena de valor esté sujeta a los lineamientos de la normativa a fin de garantizar la calidad del producto y normar el funcionamiento de las agencias certificadoras del país.

\section{Económico}

El Producto Interno Bruto (PIB), dentro de la economía ecuatoriana ha presentado un crecimiento continuo con una tasa promedio de 4.0\% entre 2000-2015 que representa un porcentaje mayor al período 1981-1999 cuando se registró un valor promedio de $2.4 \%$ (BCE, 2019). Inflación. Actualmente Ecuador tiene una inflación del $0.27 \%$ que se debe al utilizo de la deuda estadounidense, mientras la deuda interna asciende a unos \$ 58.979 millones que equivalen al 57\% de su PIB. El gobierno ecuatoriano tiene planificada la reducción del déficit fiscal de 5,64\% del PIB previsto para 2018 a 2,47\% en 2021 (BCE, 2019).

Precio caja de banano. El precio fijado por la caja de banano de 43 libras (caja 22xu) por el Ministerio de Agricultura, Ganadería, Acuacultura y Pesca, a través del Acuerdo Ministerial 135 firmado el 26 de octubre del 2018, es de \$6,30 para el año 2019; eso equivale a un aumento de 10 centavos más, respecto al que estaba vigente en el año anterior. (MAG, 2019).

Producción bananera. En Ecuador los modelos de sostenibilidad de suelos-cultivos agrícolas, implementados por el MAG en las asociaciones y fincas para cumplir con técnicas de producción orgánicas sustentables. En esta misma línea Bonisoli, Galdeano y Piedra (2018) opinan que se debe dotar a los agricultores de conocimiento e instrumentos que permitan evaluar la sostenibilidad de las plantaciones para tomar decisiones que aporten a la preservación de los ecosistemas.

La exportación bananera representa el 2\% del PIB general y aproximadamente el 35\% del PIB agrícola. Según cifras del Ministerio de Agricultura, la producción bananera de la Provincia de El Oro, representa aproximadamente el $42 \%$ de toda la producción bananera ecuatoriana. 
Desempleo. Según el INEC (2010) "Ecuador registra hasta Marzo-2019 una tasa de desempleo del 4,6\% a nivel nacional, a nivel urbano se ubicó en 5.8\%, y a nivel rural 2.2\%; las variaciones en los tres niveles no fueron significativas respecto a Marzo-2018". Respecto a marzo 2018-2019 la tasa de desempleo aumentó un 0.2\% a nivel nacional. Respecto al nivel de pobreza según el Reporte de Pobreza y Desigualdad, a Junio de 2018 el índice de pobreza se mantiene constante en $24,5 \%$ a nivel nacional. El informe también indica los niveles en las principales ciudades del país como lo son Quito, Guayaquil, Cuenca, Machala y Ambato. En todas estas ciudades ha habido un incremento en los niveles de pobreza, algunos casos de más del 2\% (INEC, 2016).

Poder Adquisitivo. La fuente ingresos a nivel nacional, se derivan gracias al empleo, representando el mayor rubro de dinero para el familiar ecuatorianas, a continuación se realiza el detalle respectivo.

Tabla. 1

Principales rubros de ingresos económicos de familias ecuatorianas

\begin{tabular}{lll}
\hline Fuentes de ingresos monetarios & Ingresos Mensuales & Porcentaje \\
\hline Ingresos provenientes del trabajo & $2.320^{\prime} 077.203$ & $83.5 \%$ \\
Renta de la propiedad y del capital & $71^{\prime} 903.425$ & $2.6 \%$ \\
Transferencias corrientes & $348^{\prime} 700.533$ & $12.5 \%$ \\
Otros ingresos corrientes & $38^{\prime} 518.661$ & $1.4 \%$ \\
Ingreso corriente monetario & $2.779^{\prime} 199.821$ & $100 \%$ \\
\hline
\end{tabular}

Fuente: elaboración propia

\section{Social}

La producción agroecológica se dirige hacia las ciudades con mayor tasa de escolaridad considerando que los consumidores de productos agroecológicos, aquellos que se ubican en la clasificación de estratos A, B y $+\mathrm{C}$, los cuales presentan un perfil con estabilidad económica, hábitos de consumo, educación superior, acceso a tecnología; definiendo de esta forma el target según su nivel de ingresos, educación y estilo de vida (INEC, 2010).

Tabla. 2 Estratificación de la población en Machala.

\begin{tabular}{lll}
\hline Niveles & \multicolumn{2}{c}{ Estratificación } \\
& Público & \% \\
\hline Alto (A) & 5.377 & 1,9 \\
Medio alto (B) & 31,700 & 11,2 \\
Medio (C+) & 64,249 & 22,7 \\
Medio-bajo (C-) & 139,537 & 49,3 \\
Bajo (D) & 42,172 & 14,9 \\
Total Población & $283,035,00$ & 100.00 \\
\hline \multicolumn{2}{c}{ Fuente: elaboración propia }
\end{tabular}

\section{Análisis de la industria}

Mediante el análisis del micro entorno se puede identificar los principales actores que intervienen en la situación de mercado. Con base a las estrategias competitivas, se establecen tres 
rutas estratégicas como liderazgo en costos, diferenciación y enfoque (O'Hare, Stewart y McColl, 2018). El estudio de la industria sirve como base en el análisis y la búsqueda de nuevas estrategias competitivas, es un modelo para evaluar cuán atractivo es la industria/mercado (Goicoechea y Souto, 2018). En base a esta herramienta y al estudio de mercado realizado para la investigación se fundamenta que:

Según datos de la entrevista realizada en los principales supermercados de la ciudad, el mercado está constituido por rivales fuertes, entre las principales marcas tenemos: Chelita, San Sebastiano, Happy Banana, Dulce Banana, otras, estas marcas comercializan un porcentaje estimado del $9 \%$ al 10\% de su producción orgánica y convencional hacia el mercado local.

El banano posee un número de intermediarios limitados, existiendo pocos mayoristas entre los cuales tenemos a los supermercados Cooperativa El Rosado, Supermercados Tia y Cooperativa la Favorita, que cuentan en algunos casos con marcas propias para la comercialización de banano y en otros poseen un número de proveedores ya establecidos.

Para la adquisición del banano orgánico, los proveedores serían los pequeños, agricultores de la fruta, que se reúnen en asociaciones de pequeños productores. Entre los sustitutos podemos encontrar las variantes de banano convencional, orito, seda, plátanos, otros. Al respecto de las barreras de entrada la más relevante son las relaciones que hay que establecer sea con los productores que con los minoristas.

\section{Matriz FODA}

Herramienta utilizada para evaluar los aspectos internos y externos de una Empresa, que afectan la misma de manera positiva o negativa (Barberán y Pazmiño, 2018). El análisis FODA es un estudio situacional de una organización en su contexto y de las características internas, a efectos que determinan sus Fortalezas, Oportunidades, Debilidades y Amenazas (Ballesteros et al, 2015).

Se realizó el detalle respectivo del banano orgánico, exponiendo como principal ventaja la compra de producción bananera de calidad a proveedores locales, como limitantes se encontró la dificultad de acceso a créditos productivos, las oportunidades son el aseguramiento de un precio oficial de banano, y en amenazas el incremento en recaudación de impuestos dentro del sector. 
Tabla. 3

Matriz de análisis FODA del producto.

Fortalezas

$>$ Accesibilidad en la compra de cajas de banano a productores o asociaciones mediante convenios.

$>$ Facilidad en el acceso de datos primarios y secundaria referente a estudios de mercado acerca de la producciòn bananera orgánica en el mercado machaleño.

Debilidades

$>$ Dificultad en la obtención de créditos bancarios, carencia de capital propio para asumir los costos del proyecto.

$>$ Carencia de maquinaria, espacio físico para el albergue y maduración de la fruta.

\section{Oportunidades}

> Precio oficial de la caja de banano orgánico establecido por el Ministerio de Agricultura y Ganadería, asegurando un valor fijo cada año por la compra del productor a las asociaciones bananeras.

$>$ Programas gubernamentales de capacitaciones y asesoría técnica en apoyo al emprendimiento productivo.

Amenazas

$>$ Aumento en la recaudación de Impuestos únicos del sector bananero provenientes de la venta local del producto.

$>$ Entrada de nuevas marcas de competidores de banano orgánico certificado.

Fuente: elaboración propia

\section{Situación de marketing actual}

Banano orgánico. Denominado musa paradisiaca de origen tropical color amarillo o verdoso, se cultiva sin pesticidas. Utiliza una variedad de nutrientes propios de origen vegetal y animal, con técnicas no contaminantes aprovechando los microambientes que favorecen la cosecha (Cabanilla, 2015). Sus propiedades son valor energético, contenido proteico y nula presencia de grasas. Posee sales minerales como hierro, fósforo y calcio, con una mayor concentración de vitaminas $\mathrm{B}, \mathrm{C}$, potasio y otros nutrientes, que aquellos producidos en sistemas con alto uso de agroquímicos (Capa, 2016). La certificación en productos orgánicos garantiza a los consumidores un producto que cumple con las normas de producción correspondientes, del mismo modo que añade valor al producto final (Bonisoli, Galdeano, Piedra y Pérez, 2019).

Distribución. Los canales de distribución sirven para poder vender y suministrar el producto, logrando aspectos de asequibilidad, poniendo al alcance del consumidor en el momento y tiempo adecuado, bajo una estrategia de distribución intensiva acaparando todos los puntos de venta señalados (Kotler y Keller, 2012).

Debido a su naturaleza perecedera se examina el tiempo y distancia que toma llevar la producción hasta el cliente final siendo factores que influyen en el precio de venta. La cadena de distribución del banano debe ser la más corta posible para reducir el costo y tiempo de entrega del producto; Los principales supermercados de la ciudad, realizan negociaciones directas con empresas bananeras sin recurrir a intermediarios, los mayoristas se abastecen de la fruta orgánica y se encargan de generar valor agregado, es decir, comercializar el producto con un empaque y etiqueta propia, generando el retorno de la inversión y la utilidad deseada (Granillo, González, Santana y Martínez, 2019). 
Producción. En el Cantón Machala según datos estadísticos del Sistema Nacional de información, existe un total de 14.756,00 Ha, destinadas al cultivo de banano convencional y orgánico, mismas que representan un $44 \%$ del hectareaje total, el estimado de cajas de banano producidas a la semana son alrededor de 3.200 por parte de pequeños agricultores locales (SIN, 2011).

De acuerdo con datos documentales del Ministerio de Agricultura y Ganadería, en la ciudad el $91 \%$ de la producción orgánica se destina hacia mercados internacionales como la Unión Europea, mientras que el 9\% de la misma es considerada como rechazo, con dicho porcentaje menor se prevé instaurar la propuesta de comercialización local, mejorando el atractivo de la fruta para la introducción en Machala. El precio de la caja oficial de banano orgánico de 45 libras, aproximadamente 105 bananas es de $\$ 9,25$, siendo el precio por unidad alrededor de \$0,20 ctvs. (MAG, 2017).

Posicionamiento. Nivel de recordación por parte del mercado en cuento a un bien o servicio específico, fundamental en las acciones de marketing empresariales para identificar el análisis competitivo frente a otros productos (Zambrano y Santana, 2014). Los factores influyen directamente en la persuasión de compra de alimentos orgánicos según el nivel de importancia son: la calidad, el empaque, las condiciones del producto (aroma, color, frescura, textura), precio, certificación, origen, marca, fecha de caducidad, ingredientes e información nutricional.

El banano se posiciona como una de los principales frutas de la canasta familiar; además de ser uno de los rubros que genera mayores ingresos a la ciudad y al País (Lazo y Hernández, 2018). Los productos orgánicos se van introduciendo en la mente de los consumidores, debido a que es asociado con bienestar y cuidado del ambiente. Los criterios de posicionamiento de la fruta para el consumidor se fundamentan en los beneficios que obtienen al consumir el producto, dentro de este punto los aspectos que posicionan el banano son la calidad, el precio y la imagen.

\section{Análisis del cliente}

Segmento. División de la empresa en una fracción menor y homogénea del mercado, identificando un grupo o grupos de personas con características similares definiendo el target (Ciribeli y Miquelito, 2015). El segmento de mercado estudiado es la población económicamente activa de la Ciudad de Machala, en general el consumidor machaleño presenta una frecuencia de compra activa. Existe una lenta tendencia en consumir productos orgánicos, es un producto con beneficios para el cuidado de la salud, la fruta está destinada para el consumo de personas con conciencia ecológica y que buscan bienestar.

Perfil del consumidor orgánico. En los últimos años se han formado diversas definiciones del consumidor orgánico, basándose en la salud y medio ambiente. Los términos pueden diferir una de la otra, sin embargo, la acepción es similar en cuanto a las características que la definen. Se presentan 3 variantes de perfil de consumidores orgánicos: 1) quienes comprar productos por factores de salud y medio ambiente; 2) quienes compran diariamente principalmente por salud; 3) por moda (López, 2019). 
Tabla. 4

Clasificación de consumidores orgánicos-no orgánicos.

\begin{tabular}{|c|c|c|c|}
\hline Hartman Group & NMI & Magrama & Descripción de categorías de consumidores \\
\hline $\begin{array}{l}\text { Consumidores } \\
\text { Core }\end{array}$ & $\begin{array}{l}\text { Consumidores } \\
\text { Lohas }\end{array}$ & $\begin{array}{l}\text { Consumidores } \\
\text { convencidos }\end{array}$ & $\begin{array}{l}\text { Involucrados e influyentes en la industria } \\
\text { orgánica; preocupados por la salud y por el } \\
\text { planeta. }\end{array}$ \\
\hline \multirow[t]{3}{*}{$\begin{array}{l}\text { Consumidores de } \\
\text { nivel medio }\end{array}$} & $\begin{array}{l}\text { Consumidores } \\
\text { naturalites }\end{array}$ & $\begin{array}{l}\text { Consumidores } \\
\text { ecologistas }\end{array}$ & $\begin{array}{l}\text { Preocupados por el medio ambiente y por hábitos } \\
\text { de consumo responsable. }\end{array}$ \\
\hline & & $\begin{array}{l}\text { Consumidores } \\
\text { preocupados por la } \\
\text { salud }\end{array}$ & $\begin{array}{l}\text { Usuarios frecuentes de productos naturales y } \\
\text { orgánicos preocupados por su salud personal y, } \\
\text { en menor medida, por el medio ambiente. }\end{array}$ \\
\hline & $\begin{array}{l}\text { Consumidores } \\
\text { drifters }\end{array}$ & $\begin{array}{l}\text { Consumidores } \\
\text { desimplicados }\end{array}$ & $\begin{array}{l}\text { Se dejan llevar por las últimas tendencias; su } \\
\text { involucramiento en el movimiento ambiental es } \\
\text { intermitente. }\end{array}$ \\
\hline \multirow[t]{3}{*}{$\begin{array}{l}\text { Consumidores } \\
\text { periféricos }\end{array}$} & $\begin{array}{l}\text { Consumidores } \\
\text { Convencionales }\end{array}$ & & $\begin{array}{l}\text { Más centrados en sí mismo; son prácticos, } \\
\text { racionales y conscientes sobre los desperdicios, } \\
\text { pero privilegian el ahorro. }\end{array}$ \\
\hline & Consumidores & & No se sienten responsables sobre el medio \\
\hline & Despreocupados & & ambiente a menos que amenace su estilo de vida. \\
\hline
\end{tabular}
Fuente: elaboración propia (basado en el criterio Hartman Group, NMI y Magram)

El género femenino de edades comprendidas desde los 25 a 60 años, con hijos preadolescentes lideran la compra y consumo de productos orgánicos, de preferencia son mujeres adultas debido a que tienen capacidad de pagar por el precio, en contraste con los consumidores más jóvenes quienes no cuentan muchas veces con un trabajo bien remunerado. La edad del cliente influye directamente en la compra, en este sentido los problemas de salud presentados motivan a preferir productos orgánicos (López, 2019).

Perfil del Público objetivo. Según la entrevista realizada en los principales supermercados de la Ciudad, quienes consumen productos agroecológicos de mayor preferencia son mujeres profesionales que en su mayoría son solteras, con un rango de edad que oscila los 20 a 45 años de edad. Tienen conciencia en apoyar de forma frecuente actividades a favor de la preservación del medio ambiente, además poseen un estilo de vida dinámico, frecuentando constantemente centro de entrenamiento como gimnasios, aeróbicos, yoga. Este tipo de consumidores pertenecen al segmento con mayor nivel de educación, poseen empleo estable y perciben sueldos mensuales superiores a un salario básico de $\$ 394,00$ (Vasco, 2015). También cuentan con una dieta alimenticia saludable constituida por productos con alto valor energético y nutricional.

Mientras que la observación directa determinó que en menor porcentaje existen hombres con edades promedio de 24 a 55 años, que compran productos saludables; caracterizándose principalmente por hacer uso de gimnasios, para mejorar su condición física e imagen, quienes por su rutina diaria, se rigen a una alimentación balanceada de alimentos con componentes naturales.

El consumidor, incluye en su alimentación productos que proporcionan beneficios a su dieta, según la observación, las personas que cumplen este perfil compran alimentos como lácteos, cereales, verduras, frutas, suplementos nutricionales, vitaminas, proteínas e incluso productos de cuidado personal; donde se destaca la compra de frutas con procedencia orgánica. 
Prefieren productos ecológicos, degradables, reutilizables, porque representa una alternativa sostenible con el medio ambiente, con noción de los beneficios que brindan para la salud y el ambiente, son respetuosos con la naturaleza y están a favor de las prácticas medioambientales promovidas por personas, organizaciones y empresas (Vasco, 2015).

\section{Estrategias de marketing}

El análisis de las estrategias de marketing se centra en aspectos internos que la empresa pueda controlar, variables controlables que la dirección pueda intervenir directamente en la respuesta del cliente/usuario (Abrigo, 2018).

Esta influencia se percibe a través de las 4Ps del marketing tradicional descritas como: producto, precio, plaza/distribución, promoción/comunicación. (Martiren y Moyano, 2018). Las estrategias aplicadas al producto y marca, presentan beneficios adicionales tales como, valor agregado, buen trato, precio, punto de venta, merchandising, calidad de la organización, capacitación, entre otros (Paladines, Campozano, Cano y Sánchez, 2017).

\section{Producto}

Agrupa atributos tales como empaque, etiquetado, precio, diseño, marca, entre otros; de tal manera que sea identificable en el mercado. Sin embargo, en Marketing esta definición se amplía para entender los beneficios que satisfagan las necesidades de los consumidores conectando con la parte emocional y psicológica del consumidor mediante la propuesta de valor Orgánico, Delicioso y Nutritivo (Heinz, Costa y Pereira, 2014).

El consumidor final prefiere un banano orgánico, nutritivo, disponible para la compra en los supermercados, además de que sea atractivo, certificado y aporte beneficios para la salud. Los atributos representativos para el diseño de la marca gráfica que informe los atributos, características y valores del producto cuales permitirán identificar al bien o servicio en el mercado y diferenciarse (Landín, Feijoo y González, 2017).

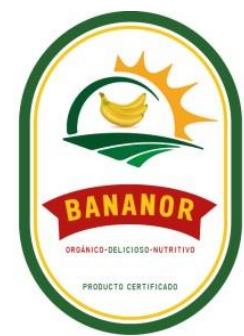

Ilustración 1. Etiqueta comercial del producto. (Fuente: elaboración propia)

En el mercado machaleño los productos sustitutos, son poco atractivos para la venta, su presentación es simple, lo cual no asegura su condición. Este tipo de empaque, es amigable con el medio ambiente, paralelamente a dicha característica, el mismo contiene las características llamativas para agilizar el proceso de compra, detallando el valor nutricional de las bananas orgánicas, para que el consumidor conozca los beneficios principales. 
Packaging. Conforme Mathon (2012) el empaque biodegradable permite mantener el almacenamiento, envasado, bodegaje y uso por parte del consumidor, pero que una vez desechado, comienza a cambiar químicamente por influencia de agentes ambientales que afectan el material. El empaque puede descomponerse en sus elementos químicos que los conforman, debido a la acción de agentes biológicos, como plantas, animales, microorganismos y hongos, bajo condiciones ambientales naturales (Añazco, Landin, Prado y Villanueva, 2018).

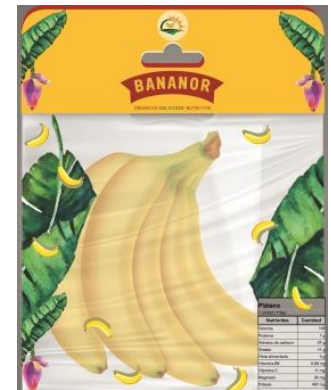

Ilustración 2. Presentación final de los empaques en perchas puntos de venta. (Fuente: elaboración propia)

\section{Precio}

El precio estimado del producto se evaluó en base a productos sustitutos, donde se determinó un costo promedio de $\$ 0,84$ ctvs. por empaque con 5 bananas, los consumidores según la encuesta realizada están dispuestos a pagar por los calidad que ofrecen las bananas; la estrategia a utilizar es pensando en un margen de rentabilidad del $20 \%$, donde se debe establecer un precio competitivo atrayendo a compradores exigentes, consiguiendo un posicionamiento adecuado y una participación de mercado aceptable (Kotler y Armstrong, 2012).

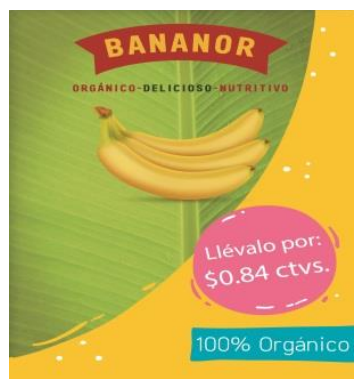

Ilustración 3. Precio final sugerido de venta al público. (Elaboración propia)

\section{Plaza}

Para la propuesta, el tipo de distribución es corta (productor - mayorista - cliente final), siendo los lugar de suministro para la asequibilidad del producto los supermercados mayoristas de la ciudad como Super Akí, Mi Comisariato y Supermaxi, con una estrategia de producto exclusiva, debido a que atrapan gran parte de las acciones de compra del público objetivo estudiado. 


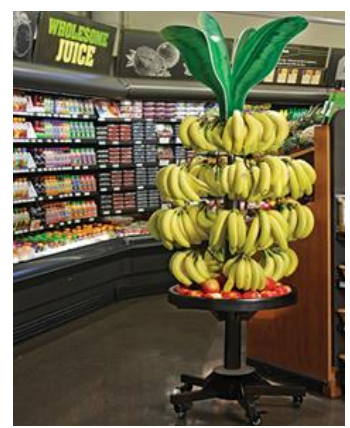

Ilustración 4. Merchandising en el punto de venta bananas. (Fuente: www.winsightgrocerybusiness.com)

\section{Promoción}

Medios offline. Ofrecen mayor audiencia pero su costo de inversión es elevado. Es acertado ejecutar esta estrategia para campañas específicas cuyo costo por impacto sea el más rentable (Marín, Terán y Lozano, 2018). Para introducir el banano orgánico en el mercado de la ciudad de Machala, se debe pautar en los siguientes medios offline: revistas especializadas de cocina, salud, agroecológicas, para captar al mercado meta del banano orgánico "Bananor"; merchandising en puntos de venta; souvenirs; vallas publicitarias; OPI's; Pantallas Led y Gigantografías (Barreto, Landín y Sánchez, 2017). El mensaje de campaña debe ser creativo, directo y específico, para comunicar la propuesta de valor, generando respuesta inmediata en el público objetivo, debido a su alto nivel de persuasión (Landín, Benítez y Moscoso, 2017).

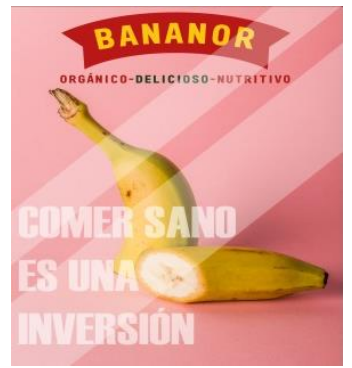

Ilustración 5. Gigantografía. (Fuente: elaboración propia)

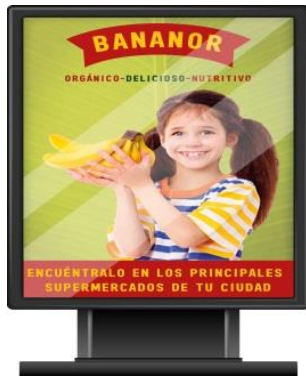

Ilustración 6. OPI.

(Fuente: elaboración propia) 


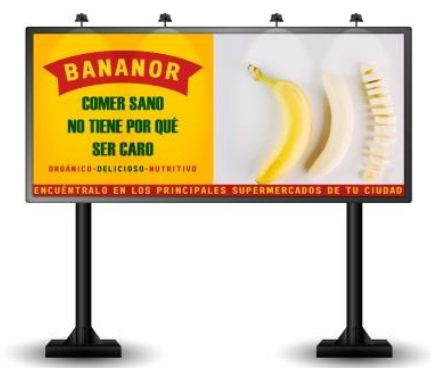

Ilustración 7. Valla publicitaria. (Fuente: elaboración propia)

Medios Online. Hoy en día el marketing por internet permite mejorar la comercialización y comunicación del producto, la social media permite interacción directa con el segmento, implementando estrategias digitales para aumentar la visibilidad y fortalecer las relaciones entre marca-usuario (Hernández y Díaz, 2016). Los medios online tienen alto grado de utilidad y su interfaz radica en la propagación de medios publicitarios vía Internet que engloba redes sociales (Facebook, Twitter, Instagram, Google+); páginas web especializadas; banners online; pop up, entre otros. Ofrecen un mayor impacto a bajo costo de inversión; además, cuentan con métricas y análisis estadísticos que permiten medir el tráfico de usuarios. Para tener mayor cobertura, se deberá utilizar dichos medios, para incrementar el alcance de las estrategias, posicionando la marca como la principal opción de banano orgánico (Landín, Benítez y Sánchez, 2017).

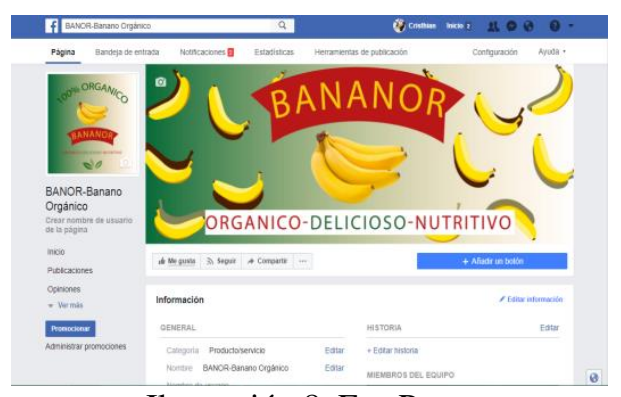

Ilustración 8. Fan Page.

(Fuente: elaboración propia)

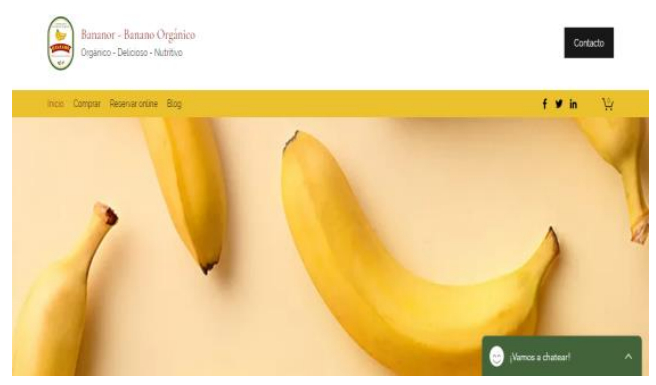

Ilustración 9. Página web.

(Fuente: elaboración propia) 


\section{Presupuesto}

Tabla. 6

Presupuesto consolidado

\begin{tabular}{lc}
\hline Medio de comunicación & Valores \\
\hline ETL (Embrace the line) & \\
Fan page & $\$ 1.000$ \\
Página Web & $\$ 1.500$ \\
ATL (Above the line) & \\
Revistas especializadas & $\$ 5.000$ \\
BTL (Below the line) & \\
Vallas publicitarias & $\$ 5.000$ \\
Gigantografías & $\$ 3.000$ \\
Opis & $\$ 4.000$ \\
Merchandising & $\$ 1.000$ \\
Subtotal & $\mathbf{\$ 2 0 . 5 0 0}$ \\
Imprevistos publicitarios 5\% & $\mathbf{\$ 1 . 0 2 5}$ \\
Total presupuesto & $\mathbf{\$ 2 1 . 5 2 5}$ \\
\hline
\end{tabular}

Fuente: elaboración propia

Para la ejecución del plan comunicacional es conveniente contar con los recursos financieros para cubrir los valores de inversión que demanda la implementación de las actividades programadas (Nieves y Fernández, 2016). En base a las estrategias publicitarias determinadas, se realizó una proforma del costo estimado del plan de marketing, donde se detalla el presupuesto general de los distintos medios de comunicación, que serán utilizados para introducir la marca, el presupuesto está proyectado para 3 meses de ejecución de campaña, con una inversión total de \$21.525 dólares.

\section{Conclusiones}

Se cumple con el objetivo de investigación de presentar un plan de marketing encaminado a ser utilizado por una empresa o asociación banera, el plan demuestra una alternativa de comercialización y consumo saludable para las mujeres de clase media-alta. Gracias al estudio se determinaron las estrategias de la mezcla de mercadeo acordes al banano, donde se identificaron las de producto, precio, plaza y promoción. Las tácticas de la propuesta direccionan correctamente hacia el objetivo, garantizando la ejecución del proyecto, en base a una correcta planificación empresarial para asegurar las metas a mediano y largo plazo.

Las marcas productoras bananeras en Ecuador, direccionan su cadena de comercialización de banano orgánico, hacia mercados extranjeros, donde las tendencias de consumo son hacia lo saludable, debido a los problemas de salud presentados en los consumidores de países desarrollados, quienes buscan el cuidado y mejora en su calidad de vida.

A nivel de la Ciudad de Machala, el consumo de productos orgánicos tiene lento crecimiento, las asociaciones productoras asignan poco porcentaje de su producción de banano orgánico (rechazo) que no cumple con estándares de calidad para sea exportada, siendo vendida hacia el mercado interno, en un precio inferior del oficial para disposición de los machaleños. 
Existe un lento crecimiento en el consumo de frutas orgánicas en Machala, los clientes están expuestos a la compra de productos llamados rechazos, en estado de descomposición, sin una presentación atractiva, que no disponen de la seguridad que pueda brindar para la salud. Sin embargo, existe un segmento de mercado en su mayoría femenino de edad adulta (20 a 45 años), profesional, con sueldo superior a un salario básico unificado, que prefiere incluir en su dieta alimenticia productos con procedencia natural. Es allí donde se evidencia la factibilidad de comercialización de banano orgánico, de calidad total, imagen, merchandising, etiquetado, empaque, que asegure las condiciones saludables de la fruta.

Haciendo énfasis en el mercado meta, el consumidor local prefiere un banano de condiciones naturales, disponible para la venta en las cadenas de supermercados dentro de la Ciudad, en presentación de empaque de plástico biodegradable. En términos generales aparte de ser un producto que aporta nutrientes, es una alternativa de consumo responsable con el medio ambiente, por las formas de cultivo del banano y por la contribución en la reducción de materiales contaminantes.

\section{Bibliografía}

Abrigo, I. (2018). Emprendimiento universitario: una oportunidad académica para traspasar $\begin{array}{llll}\text { fronteras. INNOVA } & \text { Research }\end{array}$ doi:https://doi.org/10.33890/innova.v3.n2.2018.424

Acosta, M. M., López, M. L., \& Coronel, V. C. (2018). Estrategias de Marketing para el mercado de productos orgánicos en el Ecuador. Espacios, 39(8), 29-41. Obtenido de https://www.revistaespacios.com/a18v39n08/a18v39n08p24.pdf

Alves, C., \& Pacheco, E. (2015). Gestão estratégica da qualidade para empresas produtoras de alimentos orgânicos: diretrizes para a expansão do mercado consumidor. Navus, 5(1), 51 64. Obtenido de https://dialnet.unirioja.es/servlet/articulo?codigo=5168653

Andrade, C., \& Ayaviri, D. (2018). Demanda y consumo de productos orgánicos en el Cantón Riobamba, Ecuador. Información Tecnológica, 29(4), 217-226. doi:http://dx.doi.org/10.4067/S0718-07642018000400217

Añazco, C., Landin, S., Prado, G., \& Villanueva, V. (2018). Marcador ecológico-recargable con puntero laser, alternativa para docentes, estudiantes y ejecutivos del cantón Machala. In Conference Proceedings, 2(2), 311-319. Obtenido de http://investigacion.utmachala.edu.ec/proceedings/index.php/utmach/article/view/325

Ballesteros, H., Verde, J., Costabel, M., Sangiovanni, R., Dutra, I., \& Rundie, D. \&. (2015). Análisis FODA: Fortalezas, Oportunidades, Debilidades y Amenazas. Revista uruguaya de enfermería, 5(2), 8-17. Obtenido de http://rue.fenf.edu.uy/index.php/rue/article/view/85

Barberán, L., \& Pazmiño, J. (2018). Propuesta de un plan estratégico de desarrollo turístico de la Ciudad de Manta con fines de divulgación educativo. Educare, 22(2), 45-73. Obtenido de https://dialnet.unirioja.es/servlet/articulo?codigo=6777469

Barreto, Z., Landín, S., \& Sánchez, I. (2017). Plan de Marketing de la Vieja Azul en la Provincia de El Oro. Publicaciones Utmach, 1(1), 239-243. Obtenido de http://investigacion.utmachala.edu.ec/proceedings/index.php/utmach/article/view/90/77

Benassini, M. (2009). Introducción A La Investigación De Mercados: Enfoque Para América Latina. México: Pearson Educación. 
Bonisoli, L. (2016). Análisis de los criterios de inclusión de los indicadores para la evaluación de la sostenibilidad de sistemas agrícolas. Delos, 27. Obtenido de http://www.eumed.net/rev/delos/27/criterios.html

Bonisoli, L., Galdeano, G. E., \& Piedra, M. L. (2018). Deconstructing criteria and assessment tools to build agri-sustainability indicators and support farmers' decision-making process.

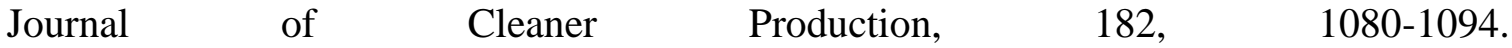
doi:https://doi.org/10.1016/j.jclepro.2018.02.055

Bonisoli, L., Galdeano, G. E., Piedra, M. L., \& Pérez, M. J. (2019). Benchmarking agri-food sustainability certifications: Evidences from applying SAFA in the Ecuadorian banana agri-system. Journal of Cleaner Production, 236. doi:https://doi.org/10.1016/j.jclepro.2019.07.054

Cabanilla Guerra, M. (2015). Empresas no tradicionales de productos alimenticios orgánicos y su gestión estratégica. Universidad y Sociedad, 10(1), 195-198. Obtenido de http://scielo.sld.cu/scielo.php?script=sci_arttext\&pid=S2218-36202018000100195

Capa, L. A. (2016). Importancia de la producción de banano orgánico. Caso: Provincia El Oro, Ecuador. Revista Universidad y Sociedad, 8(3), 64-71. Obtenido de http://scielo.sld.cu/scielo.php?script=sci_abstract\&pid=S2218$36202016000300008 \& \operatorname{lng}=$ es\&nrm=iso

Ciribeli, J., \& Miquelito, S. (2015). La segmentación del mercado por el criterio psicográfico: un ensayo teórico sobre los principales enfoques psicográficos y su relación con los criterios de comportamiento. Visión de futuro, 19(1), 33-50. Obtenido de https://www.redalyc.org/articulo.oa?id=357938586002

Estrada, M., \& Encalada, N. (2018). Producción de banano orgánico, una experiencia exitosa en La Sabana del cantón pasaje, provincia el Oro, Ecuador. Revista Científica Agroecosistemas, 5(1), 21-27. Obtenido de https://biblat.unam.mx/es/revista/agroecosistemas/articulo/produccion-de-bananoorganico-una-experiencia-exitosa-en-la-sabana-del-canton-pasaje-provincia-el-oroecuador

Ferrell, O. C., \& Hartline, M. D. (2012). Estratégia de marketing (Quinta ed.). México: Cengage Learning Editores.

García, L. M., Santillán, E. K., Blancas, N. N., \& Valenzuela, P. V. (2012). Proyecto de inversión para el desarrollo de la producción de banano orgánico ecuatoriano y su exportación a Hamburgo-Alemania. Guayaquil: Publicaciones ESPOL.

Goicoechea, C., \& Souto, J. (2018). Una aplicación del marco de las cinco fuerzas de Porter al grupo BMW. 3C Tecnología, 7(2), 10-27. Obtenido de https://www.3ciencias.com/articulos/articulo/una-aplicacion-del-marco-de-las-cincofuerzas-de-porter-al-grupo-bmw/

Granillo, R., González, I., Santana, F., \& Martínez, J. (2019). Estrategia de centros de consolidación para la distribución de tuna en México. Revista mexicana de ciencias agrícolas, 10(2), 265-276. doi:https://doi.org/10.29312/remexca.v10i2.790

Heinz, D., Costa, J., \& Pereira, E. (2014). Marketing mix in a buying network: a comparative study of the perceptions of managers and associates in a supermarket network. Revista Eletrônica de Administração (Porto Alegre), 20(2), 529-570. doi:http://dx.doi.org/10.1590/1413-2311062201238437 
Hernández, A., \& Díaz, F. (2016). El marketing digital en la clínica dental. RCOE: Revista del Ilustre Consejo General de Colegios de Odontólogos y Estomatólogos de España, 21(2), 103-109. Obtenido de https://dialnet.unirioja.es/servlet/articulo?codigo=6189115

Higuchi, A. (2015). Características de los consumidores de productos orgánicos y expansión de su oferta en Lima. Apuntes, 42(77), 57-89. Obtenido de http://www.scielo.org.pe/scielo.php?script=sci_arttext\&pid=S0252-18652015000200002

Kotler, P., \& Armstrong, G. (2012). Marketing. México D.F.: Pearson Educación.

Kotler, P., \& Keller, K. (2012). Dirección de Marketing (Decimocuarta ed.). México D.F.: Pearson Educación.

Kotler, P., Kartajaya, H., \& Setiawan, I. (2018). Marketing 4.0 Moving from traditional to digital. Madrid: Lid Editorial.

Lamas, J., Brasil, L., Correia, J., Picanço, E., \& Navarro, C. (2017). Using the MACBETH Method to improve the scenario analysis tool PESTEL in large civil construction projects. DYNA: revista de la Facultad de Minas, 84(203), 322-327. doi:http://dx.doi.org/10.15446/dyna.v84n203.65359

Landín, S., Benítez, F., \& Sánchez, I. (2017). Análisis comparativo del crecimiento y participación del consumo de cacao ecuatoriano en Europa. Conference Proceedings, $1(1)$ 1-10. Obtenido http://investigacion.utmachala.edu.ec/proceedings/index.php/utmach/article/view/232

Landín, S., Feijoo, I., \& González, F. (2017). Línea gráfica promocional y publicitaria en sectores turísticos. Caso La Tembladera. Conference Proceedings, 1(1), 230-234. Obtenido http://investigacion.utmachala.edu.ec/proceedings/index.php/utmach/article/view/89

Lazo, G., \& Hernández, Y. (2018). Estrategia de posicionamiento del Agente de Telecomunicaciones en el municipio de Pinar del Río. Avances, 21(1), 45-58. Obtenido de https://dialnet.unirioja.es/servlet/articulo?codigo $=6789910$

López, S. G. (2019). Factores que influyen en la compra de alimentos orgánicos en México. Un análisis mixto. JOURNAL: Small Business International Review, 3(2), 69-85. Obtenido de https://dialnet.unirioja.es/servlet/articulo?codigo=7007116

Marín, P., Terán, J., \& Lozano, J. (2018). La comunicación en las empresas de distribución alimentaria en España: un análisis de las herramientas online y offline. Revista de la SEECI (45), 55-73. doi:http://doi.org/10.15198/seeci.2018.45.55-73

Martiren, J. L., \& Moyano, D. (2018). La formación de mercados de alimentos en Argentina. Un análisis sobre la comercialización de las harinas de trigo entre Santa Fe y las plazas norteñas (1880-1895). América Latina En La Historia Económica, 26(1), 47-71. Obtenido de https://dialnet.unirioja.es/servlet/articulo?codigo=6762597

Mathon, Y. (2012). Envases y embalajes (Primera ed.). San Martín: Inst. Nacional de Tecnología Industrial - INTI.

Méndez, E. (2015). El Comercio Internacional de Productos Orgánicos; Retos y Desafíos. YACHANA, Revista Científica, 4, 27-36. Obtenido de http://revistas.ulvr.edu.ec/index.php/yachana/article/view/77

Nieves, G., \& Fernández, V. (2016). Un plan de marketing no es un plan de comunicación. Boletín de la Asociación Andaluza de Bibliotecarios, 31(111), 8-26. Obtenido de https://dialnet.unirioja.es/servlet/articulo?codigo $=5907060$

O'Hare, N., Stewart, A., \& McColl, J. (2018). Identifying the unique characteristics of independent fashion retailers in Scotland by utilizing Porter's generic competitive

Esta obra se comparte bajo la licencia Creative Common Atribución-No Comercial 4.0 International (CC BY-NC 4.0) 
strategy model and the marketing mix. Mediterranean Journal of Communication, 9(1), 371-386. Obtenido de https://dialnet.unirioja.es/servlet/articulo?codigo $=6254622$

Paladines, M., Campozano, M., Cano, E., \& Sánchez, C. (2017). Promoción De Productos Orgánicos Ecuatorianos Al Mercado De Milán-Italia. II Congreso Internacional En Administración De Negocios Internacionales-Ciani 2017, 613-626. Obtenido de https://dialnet.unirioja.es/servlet/articulo?codigo $=6290954$

Páramo, M. D. (2013). El proyecto de exportación, elemento básico de la planeación del marketing internacional. Revista científica Pensamiento y Gestión (34), 7-11. Obtenido de http://www.scielo.org.co/scielo.php?script=sci_arttext\&pid=S165762762013000100001

Punín, M., Martínez, A., \& Rencoret, N. (2014). Medios digitales en Ecuador perspectivas de futuro. Comunicar: Revista científica iberoamericana de comunicación y educación, 21(42), 199-207. Obtenido de http://www.redalyc.org/articulo.oa?id=15830197022

Sampieri, R. H., Collado, C. F., \& Lucio, P. B. (2010). Metodología de la Investigación. México: Mcgraw-Hill/Interamericana Editores.

Sánchez, C. J. (2017). Mercado de productos agrícolas ecológicos. Suma de negocios, 8(18), 156-163. doi:https://doi.org/10.1016/j.sumneg.2017.10.001

Sánchez, G. I., Landín, A. S., Benítez, L. F., \& Moscoso, P. A. (2017). Utilidad de los Medios de Comunicación BTL en las Universidades. Caso Universidad Técnica de Machala. Conference Proceedings, 1(1), 997-1005. Obtenido de http://investigacion.utmachala.edu.ec/proceedings/index.php/utmach/article/view/165

Spiegel, M. R., \& Stephens, L. J. (2009). Estadística (Cuarta ed.). México D.F.: McgrawHill/Interamericana Editores.

Tambaco, M. O., Alarcón, J. N., \& Alarcón, C. N. (2019). Diagnóstico situacional de los pequeños productores del banano orgánico de la provincia del Oro hacia el mercado Europeo. $\quad$ Espirales, 3(25), 60-71. Obtenido de http://www.revistaespirales.com/index.php/es/article/view/440

Torrabadella, C., \& Saiz, J. (2016). Emprendimiento y análisis PESTEL de un offshoring de servicios: Una valoración práctica. Sociedad y utopía: Revista de ciencias sociales (47), 84-101. Obtenido de https://dialnet.unirioja.es/servlet/articulo?codigo=5835307

Vasco, C., Palacios, G., \& Paspuel, S. (2015). Determinantes socioeconómicos del consumo de productos ecológicos en Quito. Siembra, 2(1), 23-28. Obtenido de https://dialnet.unirioja.es/servlet/articulo?codigo $=6140322$

Wille, H., \& Lernoud, J. (2018). The World of Organic Agriculture. IFOAM, 12-301. Obtenido de https://www.ifoam.bio/en/news/2019/02/13/world-organic-agriculture-2019

Zambrano, H., \& Santana, D. (2014). Ventajas Competitivas Del Plan De Marketing En Las Asociaciones Campesinas De Manabí Ante El Desafío De Los Mercados Globales. ECA Sinergia, $\quad 5(1), \quad 1-11 . \quad$ Obtenido de https://revistas.utm.edu.ec/index.php/ECASinergia/article/view/158/1343

Zambrano, H., Berrezueta, S., García, R., \& Alemán, R. (2017). Poblaciones de Frankliniella Parvula en lotes cultivados con banano orgánico en La Peaña, provincia El Oro, Ecuador. Revista Científica Agroecosistemas, 5(1), 86-92. Obtenido de https://aes.ucf.edu.cu/index.php/aes/article/view/145 